\title{
IL6 genotype and creatine kinase response to exercise
}

\author{
Jani Lappalainen
}

Accepted: 25 March 2009 / Published online: 8 April 2009

(C) Springer-Verlag 2009

Dear Editor,

Yamin and colleagues recently reported an association of a common single nucleotide polymorphisms (SNP) in the promoter region of interleukin-6 (IL6) gene creating a change from a $\mathrm{G}$ to $\mathrm{C}$ at position -174 , and that of tumor necrosis alpha (TNF) gene creating a change from a $\mathrm{G}$ to $\mathrm{A}$ at position -308 with systemic creatine kinase response to eccentric exercise (Yamin et al. 2008).

Previously, Olomolaiye et al. (1998) described that the presence of allele C of the IL6 SNP - 174 creates a restriction site for NlaIII, and Fishman et al. (1998) further investigated the effect of this SNP on IL6 promoter activity and also genotyped healthy Caucasian subjects and patients with juvenile chronic arthritis. However, in the paper Yamin et al. (2008) erroneously refer to this polymorphism with a NCBI reference SNP ID rs13447445, which is in fact located at position - 61 of the IL6 gene, and not at position - 174 with an ID rs 1800795 . Similarly, the TNF SNP -308 investigated by the authors should be referred with rs1800629, and not with rs 361525 which is located at position -238 .

Furthermore, the authors describe that they amplified a region for genotyping with NlaIII digestion although this genomic region contains the two IL6 SNPs (rs1800795 and rs13447445) that share a $\mathrm{G}$ to $\mathrm{C}$ transition, thus creating multiple sites for NlaIII, in addition to an internal site at location -293 to -296 . Hence this raises a concern and argues whether the relatively low frequency for genotype CC reported (0.03) (Yamin et al. 2008) can rather be explained by technical limitations of the assay or data interpretation than true characteristics of the population investigated. Fortunately, genotyping assays are available that can better overcome such potential pitfalls (Marras et al. 1999; Sylvain et al. 2004). Nevertheless, it is imperative that the authors address this for their data interpretation as it may otherwise leave the reported association of IL6 -174 SNP on eccentric exercise-associated creatine kinase response unrevealed.

\section{References}

Fishman D, Faulds G, Jeffery R, Mohamed-Ali V, Yudkin JS, Humphries S, Woo P (1998) The effect of novel polymorphisms in the interleukin-6 (IL-6) gene on IL-6 transcription and plasma IL-6 levels, and an association with systemic-onset juvenile chronic arthritis. J Clin Invest 102:1369-1376

Marras SA, Kramer FR, Tyagi S (1999) Multiplex detection of singlenucleotide variations using molecular beacons. Genet Anal 14:151-156

Olomolaiye O, Wood NA, Bidwell JL (1998) A novel NlaIII polymorphism in the human IL-6 promoter. Eur J Immunogenet 25:267

Sylvain K, Aurélie H, Marc M, Christophe R (2004) Rapid screening for HLA-B27 by a TaqMan-PCR assay using sequence-specific primers and a minor groove binder probe, a novel type of TaqMan trade mark probe. J Immunol Methods 287:179-186

Yamin C, Duarte JA, Oliveira JM, Amir O, Sagiv M, Eynon N, Sagiv M, Amir RE (2008) IL6 (-174) and TNFA (-308) promoter polymorphisms are associated with systemic creatine kinase response to eccentric exercise. Eur J Appl Physiol 104:579-586
J. Lappalainen $(\square)$

Wihuri Research Institute, Helsinki, Finland

e-mail: jani.lappalainen@helsinki.fi 